\title{
Common exotic decays of top partners
}

\section{Thomas Flacke*}

Center for Theoretical Physics of the Universe, Institute for Basic Science (IBS), Daejeon 34126,

Korea

E-mail: flacke@ibs.re.kr

\section{Nicolas Bizot}

Univ Lyon, Université Lyon 1, CNRS/IN2P3, IPNL, F-69622, Villeurbanne, France

E-mail: bizot@ipnl.in2p3.fr

\section{Giacomo Cacciapaglia}

Univ Lyon, Universit İĄe Lyon 1, CNRS/IN2P3, IPNL, F-69622, Villeurbanne, France

E-mail: g.cacciapaglia@ipnl.in2p3.fr

\begin{abstract}
Many Standard Model extensions which address the hierarchy problem contain Dirac-fermion partners of the top quark at the TeV scale. Searches for these vector-like quarks mostly focus on their decay into electroweak gauge bosons and a Standard Model quark. We discuss several classes of composite Higgs models with top partners which have underlying descriptions in terms of a fundamental gauge-fermion dynamics. All of these models contain additional BSM states which are likely to be lighter than the top partners, and which thus provide new decay channels for them. We identify the novel top partner decays which occur most commonly, provide effective Lagrangians and benchmarks, and discuss opportunities for future searches.
\end{abstract}

The 39th International Conference on High Energy Physics (ICHEP2018)

4-11 July, 2018

Seoul, Korea

${ }^{*}$ Speaker. 


\section{Introduction}

The Standard Model does not provide a dynamical mechanism for the generation of the Higgs potential which could explain why the electoweak scale is so much smaller than the Planck scale. Composite Higgs models [1] address this question by realizing the Higgs boson multiplet as a pseudo-Nambu-Goldstone boson (pNGB) multiplet of a global symmetry which is spontaneously broken by a chiral condensate which forms when an underlying BSM gauge group becomes strongly coupled in the IR (at a scale $\Lambda_{H C} \sim 1-10 \mathrm{TeV}$ ). Gauging of $S U(2) \times U(1)$ subgroup as well as explicit symmetry breaking terms (which are necessary in order to provide a top mass) generate a potential for the Higgs. Underlying models which do not contain elementary scalars whilst providing pNGBs which can be identified with the Higgs multiplet and top partners which can generate a top mass via partial compositeness have been proposed [2] and classified [3]. All of these underlying models contain a Standard Model singlet pNGB $a$ which results from an anomaly free axial $U(1)$ symmetry as well additional electroweakly charged and colored pNGBs beyond the Higgs multiplet. Table 1 shows the different electroweak and colored cosets and pNGBs which result from the underlying models. Direct searches require colored pNGBs to be heavier than $\sim 1 \mathrm{TeV}$

\begin{tabular}{|c|c|}
\hline Electroweak coset & $S U(2)_{L} \times U(1)_{Y}$ \\
\hline$S U(5) / S O(5)$ & $\mathbf{3}_{ \pm 1}+\mathbf{3}_{0}+\mathbf{2}_{ \pm 1 / 2}+\mathbf{1}_{0}$ \\
$S U(4) / S p(4)$ & $\mathbf{2}_{ \pm 1 / 2}+\mathbf{1}_{0}$ \\
$S U(4) \times S U(4)^{\prime} / S U(4)_{D}$ & $\mathbf{3}_{0}+\mathbf{2}_{ \pm 1 / 2}+\mathbf{2}^{\prime}{ }_{ \pm 1 / 2}+\mathbf{1}_{ \pm 1}+\mathbf{1}_{0}+\mathbf{1}^{\prime}$ \\
\hline \hline Color coset & $S U(3)_{c} \times U(1)_{Y}$ \\
\hline$S U(6) / S O(6)$ & $\mathbf{8}_{0}+\mathbf{6}_{(-2 / 3 \text { or } 4 / 3)}+\overline{\mathbf{6}}_{(2 / 3 \text { or }-4 / 3)}$ \\
$S U(6) / S p(6)$ & $\mathbf{8}_{0}+\mathbf{3}_{2 / 3}+\overline{\mathbf{3}}_{-2 / 3}$ \\
$S U(3) \times S U(3)^{\prime} / S U(3)_{D}$ & $\mathbf{8}_{0}$ \\
\hline
\end{tabular}

Table 1: The SM quantum numbers of the pNGBs appearing in underlying composite Higgs models [5].

$[4,5]$, while the SM singlet $a$ is being tested by current LHC resonance searches $[5,6,7]$ and particularly unconstrained in a mass window $15 \mathrm{GeV}<m_{a}<65 \mathrm{GeV}$ [8].

\section{Top-partners and common exotic decays}

Vector-like top partners (VLQs) are searched for by the ATLAS and CMS collaborations. Most searches focus on pair production of charge 5/3 (X), 2/3 (T), -1/3 (B), and -4/3 (Y) VLQs through their QCD interaction with subsequent decay into an electroweak boson $(W, Z, h)$ and a 3 rd generation quark $(t, b)$. Assuming the absence of other decay channels, the current bounds on VLQ masses lie in the regime of $1.0-1.4 \mathrm{TeV}$, depending on the VLQ charge and branching ratios. ${ }^{12}$

\footnotetext{
${ }^{1}$ See Ref. [9] for a recent combination of ATLAS VLQ searches. CMS bounds from individual searches are comparable. At the time, a combination of CMS bounds is not yet available.

${ }^{2}$ Searches for single production of VLQs (see e.g. Refs. [10, 11, 12]) and VLQs decaying to electroweak bosons and a light quark (see e.g. Refs. $[13,14,15,16]$ ) have additional discovery potential, but are more model-dependent, and less covered by experimental searches at this time.
} 
Additional pNGBs beyond the Higgs multiplet (which are present in all underlying models discussed) are naturally lighter than the VLQs and thus allow for additional VLQ decay channels. A given underlying model determines the quantum numbers and couplings of the pNGBs to SM particles, as well as the multiplet structure of potential top partners. The couplings of top partners to pNGBs and 3rd generation quarks arise through explicit symmetry breaking terms which determine the mixing of the top with top partners. As all pNGBs (the Higgs multiplet and BSM pNGBs) arise from the Goldstone boson matrix, the couplings of top partners to electroweak bosons vs. new pNGBs are related through a small number of underlying parameters. Thus, in a given underlying model, the VLQ decay channels and relations of branching ratios are determined [17]. Scanning through the different underlying models we searched for "common exotic top partner decays", i.e. novel decays which are present several models, and which have branching ratios comparable to the "standard" decays into electroweak gauge bosons and 3rd generation quarks. We found several cases:

- singlet pseudo-scalar $(a): T \rightarrow t a$ and $B \rightarrow b a$, occuring in all models,

- exclusive pNGB $(\eta): T \rightarrow t \eta$, where $T, \eta$ are odd under an approximate parity (broken only by Wess-Zumino-Witten terms) in models with $S U(4) / S p(4)$ electroweak coset,

- colored pNGB $\left(\pi_{6}\right): X \rightarrow \bar{b} \pi_{6} \rightarrow \bar{b} t t$ in models with $S U(6) / S O(6)$ color coset, and

- charged pNGBs $\left(\phi^{+}, \phi^{++}\right): X \rightarrow t \phi^{+}$and $X \rightarrow b \phi^{++}$, in models with $S U(5) / S O(5)$ electroweak coset.

Effective Lagrangians and benchmark parameters which arise from underlying models are provided in Ref. [17]. In all cases, VLQs can be produced in pairs through QCD interactions, and branching ratios of the exotics decays are of the same order as "standard" decays. In the case of $T \rightarrow t \eta$, this is even the sole decay channel, as standard decays are forbidden by an approximate parity. All these exotic decays yield a third generation quark and two electroweak bosons, gluons, or fermions in the final state. As an example for the multitude of new final states, we show the branching ratios of $T$ and $a$ as a function of $m_{a}$ in Benchmark model 1 in Fig.1.
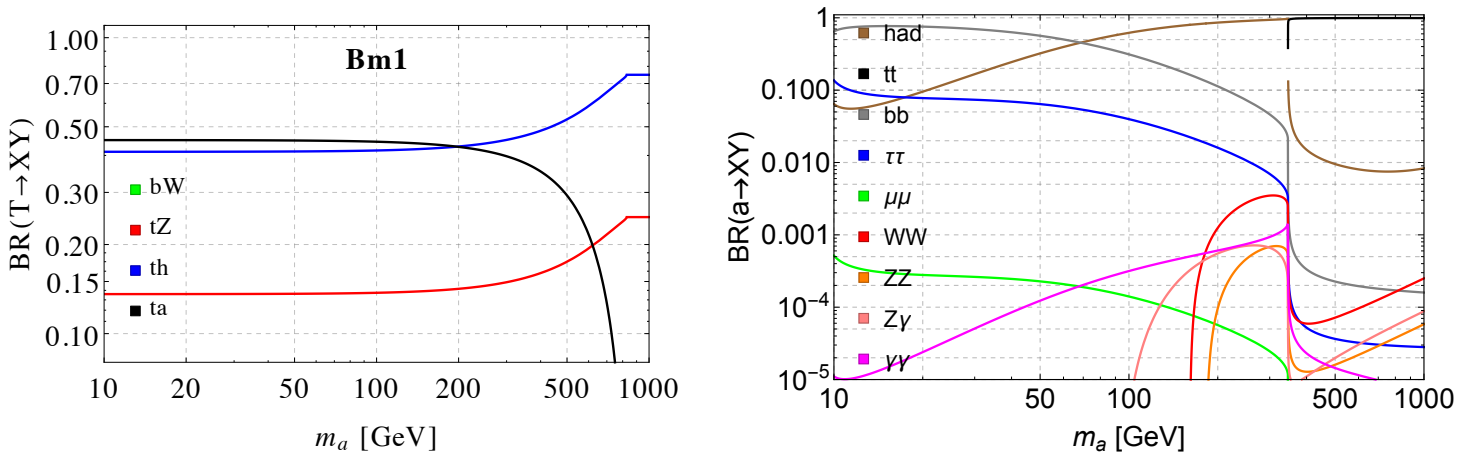

Figure 1: Left: Branching ratios of $T$ as a function of the mass $m_{a}$ in the benchmark model Bm1. Right: Branching ratios of $a$ as a function of the mass $m_{a}$ in the benchmark model Bm1. See Ref. [17] for full benchmark specifications.

The final states typically do not involve large missing energy (which results from leptonic $W$ or invisible $Z$ decays, only), and are signified by a large multiplicity of high $p_{T}$ objects. Thus the 
final states are a priori fully reconstrucable and distinguishable from Standard Model background if dedicated searches are performed. Existing VLQ pair production searches as well as R-parity violating SUSY searches are potentially sensitive to these final states, but the determination of efficiencies and resulting bounds require recasts of these searches, which are in progress. Furthermore, all cases apart from the exclusive pNGB $\eta$ allow for single-production of the top partner with subsequent exotic decay, for which the same qualitative features apply.

\section{Conclusions}

Composite Higgs Models provide a viable solution to the hierarchy problem but they still pose many challenges and leave room for exploration in theory and model-building. Effective field theory descriptions of composite Higgs models are only part of the story. Underlying models predict additional pNGBs which lead to novel BSM LHC signatures. We studied and summarized bounds various pNGBs from direct searches. Decays of top partners to 3rd generation quarks and new pNGBs occur commonly in underlying theories of composite Higgs. These decays lead to many final states which are not targeted by current LHC searches, and need to be studied in more detail.

Acknowledgements: This work was supported by IBS under the project code, IBS-R018-D1.

\section{References}

[1] D. B. Kaplan and H. Georgi, Phys. Lett. 136B (1984) 183.

[2] J. Barnard, T. Gherghetta and T. S. Ray, JHEP 1402 (2014) 002

[3] G. Ferretti and D. Karateev, JHEP 1403 (2014) 077

[4] G. Cacciapaglia, H. Cai, A. Deandrea, T. Flacke, S. J. Lee and A. Parolini, JHEP 1511 (2015) 201

[5] A. Belyaev et al., JHEP 1701 (2017) 094, Erratum: [JHEP 1712 (2017) 088]

[6] H. Cai, T. Flacke and M. Lespinasse, arXiv:1512.04508 [hep-ph].

[7] A. Belyaev et al., Phys. Rev. D 94 (2016) no.1, 015004

[8] G. Cacciapaglia, G. Ferretti, T. Flacke and H. Serôdio, Eur. Phys. J. C 78 (2018) no.9, 724

[9] M. Aaboud et al. [ATLAS Collaboration], arXiv:1808.02343 [hep-ex].

[10] M. Backović, T. Flacke, S. J. Lee and G. Perez, JHEP 1509 (2015) 022

[11] M. Backović, T. Flacke, J. H. Kim and S. J. Lee, Phys. Rev. D 92 (2015) no.1, 011701

[12] M. Backović, T. Flacke, J. H. Kim and S. J. Lee, JHEP 1604 (2016) 014

[13] C. Delaunay, T. Flacke, J. Gonzalez-Fraile, S. J. Lee, G. Panico and G. Perez, JHEP 1402 (2014) 055

[14] T. Flacke, J. H. Kim, S. J. Lee and S. H. Lim, JHEP 1405 (2014) 123

[15] M. Backović, T. Flacke, J. H. Kim and S. J. Lee, JHEP 1504 (2015) 082

[16] G. Cacciapaglia et al., JHEP 1707 (2017) 005

[17] N. Bizot, G. Cacciapaglia and T. Flacke, JHEP 1806 (2018) 065 